\title{
Research Paper: \\ The Lower Levels of Serum and RBC Cholinesterase in Acute Opioid Poisoning
}

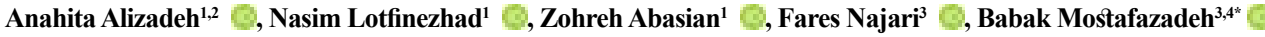 \\ 1. Department of Clinical Toxicology, Faculty of Medicine, Mashhad University of Medical Sciences, Mashhad, Iran. \\ 2. Medical Toxicology Research Center, Mashhad University of Medical Sciences, Mashhad, Iran. \\ 3. Department of Forensic Medicine and Toxicology, Faculty of Medicine, Shahid Beheshti University of Medical Sciences, Tehran, Iran \\ 4. Toxicological Research Center, Shahid Beheshti University of Medical Sciences, Tehran, Iran.
}

\begin{tabular}{|l|l|}
\hline $\begin{array}{l}\text { Use yourdevice toscan } \\
\text { and read the article online }\end{array}$ & $\begin{array}{l}\text { How to cite this paper Alizadeh A, Lotfinezhad N,Abasian Z, Najari F, Mostafazadeh B. The Lower Levels of Serum and } \\
\text { RBC Cholinesterase in Acute Opioid Poisoning. Iranian Journal of Toxicology. 2020; 14(2):111-114. }\end{array}$ \\
def http://dx.doi.org/10.32598/ijt.14.2.672
\end{tabular}

\section{(a) $0(3$}

Article info:

Received: 02 Jan 2020

Accepted: 13 Feb 2020

Online Published: 01 Apr 2020

* Corresponding author:

Babak Mostafazadeh, MD.

Address: Toxicological Research Center, Shahid Beheshti University of Medical Sciences, Tehran, Iran.

E-mail:mstzbmd@sbmu.ac.ir

\section{ABSTRACT}

Background: Cholinesterase is an enzyme that plays a critical role in regulating neural transmission. Many factors may decrease the cholinesterase levels in serum and Red Blood Cells (RBCs). It is suggested that the use of opium may effectively change the levels of Cholinesterase in serum and RBCs. This study aimed to evaluate the serum and RBC levels of cholinesterase in patients who were acutely poisoned with opium.

Methods: This cross-sectional study was conducted in 65 patients suffering from acute opium poisoning. The serum levels of cholinesterase were measured using an automated analyzer. The levels were also measured in RBCs, using a colorimetric method. Simultaneously, 65 patients served as the positive controls from among those poisoned with organophosphates.

Results: The serum cholinesterase levels were lower than the normal range in $16.9 \%$ of patients poisoned with opium and in $76.9 \%$ of those poisoned with organophosphate agents $(\mathrm{P}<0.001)$. Similarly, the levels of RBC cholinesterase were lower than the normal range as found in $64.6 \%$ and $15.4 \%$ of patients poisoned acutely with opium (Group 1) and organophosphate (Group 2), respectively $(\mathrm{P}<0.001)$.

Conclusion: Acute opium poisoning effectively reduced both the levels and activities of cholinesterase in the patients' serum and RBCs significantly different from those noted in patients suffering from organophosphate toxicity.

Keywords: Acute poisoning; Cholinesterase levels; Serum; RBCs; Opium; Organophosphates

\section{Introduction}

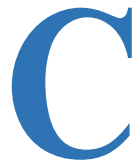

holinesterase is an important enzyme in the human body involved in the metabolism of many biochemical reactions. The function and normal levels of this enzyme are essential for the operation of many systems, including the proper activities of neurotransmitters, and the metabolism of some drugs and chemicals, including succinylcholine and organophosphate agents. Additionally, mus- cle relaxants, especially succinylcholine, should be used for some treatment plans, such as tracheal intubation. In fact, the normal level and function of cholinesterase is essential for the drug metabolism and restoration of muscle contraction. Also, according to valid sources, health conditions, such as liver, kidney and genetic disorders, trauma, malnutrition, anemia, toxins and chemical agents, consumption of substances, such as morphine and codeine, may reduce the cholinesterase activity or level $[1,2]$. 
Considering that the consumption of opium and its derivatives is relatively common in Iran [3], the aim of this study was to evaluate the serum and red blood cells (RBCs) levels of cholinesterase in patients who were acutely poisoned with opium.

\section{Materials and Methods}

Study Participants: This cross-sectional study was conducted on 65 patients suffering from acute toxicity with opium (Group 1) who were referred to and hospitalized in the poisoning ward of Imam Reza Hospital in Mashhad during the winter of 2018. Simultaneously, 65 patients with organophosphate poisoning were selected as positive controls (Group 2). The second group consisted of symptomatic patients who had been poisoned with a variety of organophosphate agents that was expected to decrease the levels of cholinesterase in both the serum and RBCs.

Inclusion Criteria: In the experimental group (Group 1), the definitive diagnosis of opium-related poisoning was confirmed by the urinalysis for each patient. The study method and concept were explained to the patients, and they were included in the study after they agreed to participate and completed the informed consent form.

Exclusion Criteria: Patients with any of the following conditions were excluded from the study, due to the possibility of interference with their cholinesterase response:

History of liver diseases, hemoglobinopathy, drug dependence, anemia (hemoglobin $<10 \mathrm{~g} / \mathrm{dL}$ ), having had a blood transfusion within the past 30 days, prolonged occupational exposure to chemicals, using drugs that inhibit cholinesterase activity, or having a history of Alzheimer or Myasthenia gravis.

Blood Analysis: Within the first 12-24 hours of hospitalization, $5 \mathrm{ml}$ of non-heparinized blood sample was taken from each patient. Approximately 1-1.5 mL of the blood was collected in a tube containing citrated anticoagulant for the purpose of measuring the serum and RBCs cholinesterase levels at the reference laboratory. The serum cholinesterase was measured using an automated analyzer 3000BT (Italy). The level of RBC cholinesterase was also measured colorimetrically, using a Perkin-Elmer spectrophotometer (USA) and Fars Biorex kits. The normal level of cholinesterase in serum was considered within the range of 4000 7000 units/L.

The study's objective was to determine the levels of cholinesterase in patients suffering from acute opium toxicity. The results were summarized by stating the absolute fre- quencies and percentages of categorical variables, examined through chi-square test. The data were analyzed using SPSS V. 16. A P-value of 0.05 or less was considered a statistically significant difference.

\section{Results}

In this study, 65 patients with acute opium toxicity (Group 1) and 65 other patients (Group 2) suffering from organophosphate poisoning were assessed. In both groups, the patients' age ranged from 18 to 65 years old. Regarding patients' gender distribution, 32 patients $(49.2 \%)$ and 57 patients $(87.6 \%)$ were male in groups 1 and 2 , respectively $(\mathrm{P}=0.040)$. The remaining individuals in each group were females. The serum cholinesterase levels were lower than the normal range in $16.9 \%$ of patients poisoned with opium and $76.9 \%$ of those poisoned with organophosphate agents, revealing a significant difference between the two groups $(\mathrm{P}<0.001)$. Similarly, the levels of RBC cholinesterase were lower than the normal range in $64.6 \%$ and $15.4 \%$ in groups 1 and 2 , respectively, indicating a significant difference between the groups $(\mathrm{P}<0.001)$.

\section{Discussion}

Cholinesterase plays a critical role in regulating the transmission of neural signals. This enzyme has two main isoenzymes, i.e., $C$ cholinesterase, which is mainly present in the nervous system and RBC membrane and butyryl cholinesterase that is found in plasma, liver, pancreas, and brain. Normal levels of acetyl cholinesterase in human RBC range from $5-10 \mathrm{U} / \mathrm{mL}$ or $30-40 \mathrm{U} / \mathrm{g}$ of the hemoglobin. In serum, the normal cholinesterase levels range between 8 and $18 \mathrm{U} / \mathrm{mL}$ [4].

One of the important roles of cholinesterase is to metabolize drugs used to relax skeletal muscles. This is especially important for the metabolism of a depolarizing relaxant called succinylcholine [5]. Succinylcholine is used in the early stages of anesthesia in preparation to intubate patients, and sometimes in critically ill patients requiring emergency intubation [5]. If for any reason, the amount or activity of cholinesterase is low, muscle relaxation or paralysis caused by succinylcholine will persist for a long time and the anesthetist may have difficulty restoring the patient's muscle strength to normal.

Therefore, it is important to be aware of the patient's cholinesterase level and the responses to the test for the underlying conditions, such as drug usage $[5,6]$. Therefore, it is necessary to check both cholinesterase and dibucaine concentrations before using succinylcholine. 
At the lower values of these variables, the risk of longterm apnea following the use of succinylcholine may be raised. It is worth noting that the the dibucaine concentration is used to differentiate individuals who have substitution mutations of the butyryl cholinesterase resulting in lower enzyme activity.

According to valid sources [1, 2], many factors may reduce the cholinesterase levels such as anemia, hemoglobinopathies, anti-malarial drugs, liver diseases, malnutrition, and pregnancy. Also, genetic factors and the use of certain drugs such as morphine, codeine, succinylcholine, and some toxins, such as organophosphates may lower the butyryl cholinesterase activity [1, 2]. Some studies have shown that in cases of inflammatory disorders and in critically ill patients admitted to the intensive care units, this enzyme has been reduced and hence its use as a prognostic agent $[7,8]$. The levels of cholinesterase in serum and RBCs can be used for monitoring organophosphate poisoning.

However, its level may not be closely related to the severity of the poisoning [9]. For the reasons stated, it is necessary to measure the patient's serum cholinesterase levels and verify the interpretation before deciding to use such drugs, as succinylcholine for emergency intubation or in cases of toxicity analysis. For this purpose, this study selected a group of patients who were poisoned with organophosphate agents as the positive controls (Group 2). The experimental group was chosen from those with acute opium poisoning (Group 1) to determine differences in the levels of cholinesterase in acutely poisoned patients.

Our results showed lower than normal serum levels of cholinesterase in $16.9 \%$ of patients poisoned with opium and $76.9 \%$ of those poisoned with organophosphate. Particular attention must be paid to this important phenomenon when taking action to induce anesthesia or perform surgery in those who have used some of these agents or when trying to identify potential drugs or organophosphate agent as the cause of poisoning. Of note, the results of this study showed that in $15-16 \%$ of people who used any of these drugs may have decreased cholinesterase levels and thus precautions must be taken before using Succinylcholine. Also in people who are tested for a variety of toxicities by assessing cholinesterase levels that the low levels of this enzyme are confirmed, exposure to other toxins, such as organophosphate should also be ruled out.

Numerous studies have investigated the effects of opioids on the level of acetyl cholinesterase. Opioids have been considered as modulators of the cholinergic system since the early 1970's when morphine was found to impair acetylcholine release and to facilitate neurotransmitter activities in the neuromuscular junction $[10,11]$. In this study, no association was found between serum acetyl cholinesterase and the total period of use or average daily opioid intake. Patients who use opioids chronically may suffer from malnutrition, which in turn can contribute to lower acetyl cholinesterase levels.

In 2017, two scientists studied the effects of morphine and tramadol on the acetyl cholinesterase activity [12]. They showed that the enzyme activity in the cerebral cortex increased following the administration of repeated doses of tramadol or morphine. The data showed that the two drugs had a significant effect on the acetyl cholinesterase activity [12]. Bailey and Briggs in an in vitro study examined the effect of 17 commonly used substances on serum's pseudo-cholinesterase activity [13].

In the present study, morphine, quinidine and thioridazine at therapeutic doses could inhibit pseudo-cholinesterase activity higher than $5 \%$. It was also concluded that the higher concentrations of these substances may have a higher inhibitory effect. Therefore, in this study, the in vivo evaluation of the conditions in humans at higher doses was recommended [13]. Our findings indicated that cholinesterase levels may also be lower in acute intoxicated patients. On the other hand, more studies in larger sample sizes are warranted to examine the effect of drug use, the type of drug, and other baseline variables, such as age, sex, nutritional status, and pharmacokinetic differences on the level of cholinesterase

\section{Conclusion}

Serum cholinesterase level was lower than the normal range in $16.9 \%$ of patients with acute opium poisoning. Also, the level of this enzyme was reduced significantly in RBCs of $64.6 \%$ of these patients. Acute opioid poisoning can be reliably monitored by the measurement of serum and RBC cholinesterase levels. Broader studies are recommended to increase the validity and accuracy of the results, and to investigate the associated factors and differences involved in acute and chronic opioid poisoning.

\section{Ethical Considerations}

\section{Compliance with ethical guidelines}

All authors met the criteria of authorship based on the recommendations of the international committee of medical journal editors. 


\section{Funding}

This research did not receive any specific grant from funding agencies in the public, commercial, or not-forprofit sectors.

\section{Author's contributions}

All authors contributed in preparing this article.

\section{Conflict of interest}

The authors declared no conflict of interest.

\section{Acknowledgments}

The authors express appreciation to the Department of Clinical Toxicology, Imam Reza Hospital, Mashhad University of Medical Sciences, Mashhad, Iran.

\section{References}

[1] Katewa SD, Katyare SS. Antimalarials inhibit human erythrocyte membrane acetylcholinesterase. Drug Chem Toxicol 2005; 28(4):467-82. [DOI:10.1080/01480540500262912] [PMID]

[2] Eddleston M. Insecticides: Organic phosphorus compounds and carbamates. In: Hoffman RS, Nelson LS, Goldfrank LR, Howland MA, Lewin NA, Smith SW. Goldfrank's Toxicologic Emergencies. 11th ed. New York: McGraw-Hil Education; 2019. 1494-500. https://books.google.com/ books?id=a6QwswEACAAJ\&dq

[3] Ekhtiari H. Addiction studies in Iran; neuroscientists need to do more. Basic Clin Neurosci. 2010; 1(3):3-4. http:/ / bcn.iums ac.ir/article-1-31-en.html

[4] Guo CJ, Sutin KM. Neuromuscular blockers. In: Hoffman RS, Nelson LS, Goldfrank LR, Howland MA, Lewin NA Smith SW. Goldfrank's Toxicologic Emergencies. $11^{\text {th }} \mathrm{ed}$. New York: McGraw-Hill Education; 2019. https://books. google.com/books?id=a6QwswEACAAJ\&dq

[5] Sener EB, Ustun E, Kocamanoglu S, Tur A. Prolonged apnea following succinylcholine administration in undiagnosed acute organophosphate poisoning. Acta Anaesthesiol Scand. 2002; 46(8):1046-8. [DOI:10.1034/j.1399-6576.2002.460821.x] [PMID]

[6] Rezaei M, Zolhavarieh SM, Jalalvand H, Hamzehei R. [Pseudo cholinesterase enzyme deficiency: Two rare cases and treatment (Persian)]. J Res Urol. 2017; 2(1):23-8. [DOI:10.30699/ acadpub.jru.2.1.23]

[7] Zivkovic AR, Bender J, Brenner T, Hofer S, Schmidt K. Reduced butyrylcholinesterase activity is an early indicator of trauma-induced acute systemic inflammatory response. J Inflamm Res. 2016; 9:221-30. [DOI:10.2147/JIR.S117590] [PMID] [PMCID]

[8] Zivkovic AR, Schmidt K, Stein T, Münzberg M, Brenner T. Bedside-measurement of serum cholinesterase activity pre- dicts patient morbidity and length of the intensive care unit stay following major traumatic injury. Sci Rep. 2019; 9:10437. [DOI:10.1038/s41598-019-46995-y] [PMID] [PMCID]

[9] Aygun D, Doganay Z, Altintop L, Guven H, Onar M, Deniz $\mathrm{T}$, et al. Serum acetylcholinesterase and prognosis of acute organophosphate poisoning. J Toxicol Clin Toxicol. 2002; 40(7):903-10. [DOI:10.1081/CLT-120016962] [PMID]

[10] Motel WC, Coop A, Cunningham CW. Cholinergic modulation by opioid receptor ligands: Potential application to Alzheimer's disease. Mini Rev Med Chem. 2013; 13(3):456-66. [D OI:10.2174/1389557511313030012] [PMID] [PMCID]

[11] Haghpanah T, Afarinesh MR, Divsalar K. A review on hematological factors in opioid-dependent people (opium and heroin) after the withdrawal period. Addict Health. 2010; 2(12):9-16. [PMID] [PMCID]

[12] Elwy AEM, TablG. Impact of tramadol and morphine abuse on the activities of acetylcholine esterase, $\mathrm{Na}+/ \mathrm{K}+-\mathrm{ATPase}$ and related parameters in cerebral cortices of male adult rats. Electron Physician. 2017; 9(3):4027-34. [DOI:10.19082/4027] [PMID] [PMCID]

[13] Bailey DN, Briggs JR. Studies of the inhibition of serum pseudocholinesterase activity in vitro by commonly used drugs. Am J Clin Pathol. 2005; 124(2):226-8. [DOI:10.1309/9X177KVU1TMMAX28] 